\title{
Indices
}

\section{Index locorum}

\section{a) sacrae scripturae}

Genesis

1,1: 399

2, 8: 227

3, 7: 227

3, 21: 227

19, 30-38

22, 10: 30

Deuteronomium

$$
\text { 6, 5: } 27
$$

Iudicum

9,14: 245

9,14-15: 228

Ruth

3, 7-14: 28

Regnorum I

1, 11: 30

Maccabaeorum II

7, 20-23: 30

14, 4: 228

Psalmi

7, 16: 233

17, 34: 243

$34,2: 27$

41, 5: 37

43, 22: 36

57, $5: 250$

58,4: 248,250

58, 15: 243

60, 9: 27

$86,7: 37$

90, 5: 37

91, 13: 228

101, 10: 34

108, 13: 398
117, 12: 254

118 (119), 62: 28

124 (125), 1: 27

125, 5-6: 230

126, 2: 34

139, $4: 250$

140, 2: 35,49

146 (147), $5: 33$

146, 9: 248

Proverbia

24, $54(30,10): 250$

26, 27: 233

Ecclesiastes (Jesus Sirach)

3, 7: 26

10, 11: 249

27, 7: 26

Canticum

5, 10-15: 159

Sapientia Salomonis

$3,1: 27$

Iob

4, 11: 55

5, 25: 226

14, 9: 228

21, 18: 228

29, 18: 228

40, 25: 250

Osee

11, 10: 236

Michaeas

4, 3: 228

5, 7: 236

Sophonias

16, 5: 250 
Isaias

10, 14: 235,246

11, 6: 228

14, 29: 250

27, 1: 249

65, 25: 228

Ieremias

12, 1-2: 232

12, 4: 232

38 (31), 29: 228

Ezechiel

18, 2: 228

Matthaeus

5, 10: 34

5, 15: 394

6, 19-20: 255

7, 15: 236

10, 34: 30

10, 36: 32

17, 20: 228

23, 24: 244

23, 37: 246

25, 26: 230

Marcus

3, 17: 410

4, 21:394, 395

Lucas

8, 16: 394
11, 33: 394, 399

15, 4: 238

24, 32: 27

Johannes

5, 11: 397

10, 12: 238

Ad Romanos

12, 24: 233

16, 3: 34, 38

Ad Corinthios I

16, 13: 33

Ad Corinthios II

12, 7: 226

Ad Ephesios

2, 2: 37

$6,17: 30$

6, 19: 397

Ad Hebraeos

11, 25: 238

Epistula Jacobi

5, 11: 397

Epistulae Petri

I 4, 10: 396

Apocalypsis

6, 1-2: 135

6, 5-6: 133

6, 8: 130

12, 9: 250

19, 11-16: 135 


\section{b) aliorum scriptorum}

Acacius

136-137: 262

186-189: 262

231-235: 267

236-243: 267

259: 272

261: 273

261-266: 262

Achilles Tatius

Leuc. et Clitophon 1, 11: 374

1,17: 362

5, 5: 367

Aelianus

De anim. 2, 13: 249

Aesopus

fab. $62: 251$

136: 243

199: 236

267: 254

Ammianus Marcellinus

XVII 7, 1-2: 141

XVII 7, 1-8: 151

Anaximenes

Rhet. I 4, 1421 b25: 109

I 4, 1421b-32: 109

Anna Comnena

Alexias I 1, 2: 108

I 2, 2: 106

I 2, 3: 100

V 7, 3: 374

VI 13, 1: 374

X 3, 6: 374

$\mathrm{X} 5,10: 374$

XII 5, 1: 374

Anonymus de re strat. XI 1-9: 135

Anthologia Palatina

9, 449: 365

Antoninus Liberalis

Metamorph. Syn. (Papathomopulos)

34: 364

Aphthonius

Progymn. 2-3: 360

Progymn. 34-36: 360
Progymn. 37: 467

Arethas

or. 57 (1-6): 259

or. $57(1,4-2,27)$ : 265

or. $57(3,2-4,2): 265$

or. $57(4,32-6,9): 265$

or. 58 (7-10): 259

or. $58(9,22-27): 268$

or. 59 (11-16): 259

or. 61 (23-30): 259

or. $61(23,1-13): 272$

or. $61(24,5-8): 273$

or. $61(24,27-25,9): 271$

or. $61(28,25-29,7): 270$

or. $61(45,15-16): 273$

or. 62 (31-34): 259

or. $62(31,3-8): 268$

or. 62 (32, 19-22): 269

or. $63(35,3-18)$ : 262

or. $63(36,23-28)$ : 263

or. $63(37,13-38,22): 262$

or. 64 (39-42): 259

or. $64(39,4-40,14)$ : 266

or. $64(40,15-41,17): 266$

or. $64(41,18-42,8): 267$

or. 65 (43-48): 259

or. 65 (46, 9-10): 271

Scripta minora 187,11 ss.: 416

Aristides

$18(8,15-9,3): 145$

$(8,16): 155$

$(9,3-4): 145$

$(9,4-10,12): 145$

$(9,9-10): 149$

$(9,12-16): 149$

$(9,16-17): 149$

$(9,18-19): 149$

$(9,22-23): 154$

$(10,12-11,16): 146$

$(10,13): 155$

(10, 17-18): 146

$(10,18-11,2): 146$

$(10,21-11,1): 152$ 


$$
\begin{aligned}
& (11,2-10): 146 \\
& (11,4): 154 \\
& (11,6-7): 150-152 \\
& (11,10 \text { ss.): } 146 \\
& (11,12-13): 154 \\
& (11,17-23): 146 \\
& (11,21): 155 \\
& (11,23-26): 146
\end{aligned}
$$

Aristophanes

equites 523: 253

nubes 144-147: 254

997: 229

ranae 289: 226

Aristoteles

Nic. Eth. 1108a27-28: 361

1098b: 434

Rhet. 1358b 23-26: 109

1359b 18-23: 109

1359b 28-32: 109

Athenaeus

229a: 244

Ausonius

Epitaph. (carm. 15, 4): 194

Basilius

in leg. gent. lib.(ed. Wilson)

I 8-10: 290

I 27: 290

II $30-31$

II 37-38: 290, 291

IV 5-11: 291

IV 19-28: 291

IV 28: 291

IV 30-31: 291

V 26-27: 290

V 31-35: 291

V 47-54

VII 2: 292

VII 23-30: 291

VII 39: 291

VIII 2-3: 290

IX 91

in XL martyros PG 31, 508-525:

173-174

Cato

disticha 34: 195
Cicero

Cluent. 31: 194

Inv. 1, 101: 200

Tull. 8: 200

Tusc. 2, 61: 194

Verr. 5.33.86: 469

Codex Theodosianus

XIII 3, 5: 292

XIV 1, 1: 292

Constantinus Manasses

Aristarchus et Callithea 95, 1: 363

Constantinus Porphyrogenitus

de cer. I 28: 270

de cer. I 36 (27): 267

de cer. I 37 (28): 264

de cer. I 96 (438, 3-440, 1): 121, 124

de cer. II 10 (545-546): 267

de cer. II 14: 264

de sent. 110, 27-30: 118

234, 14-27: 118

relatio de legationibus 3: 123

tres tractatus 144, 792-794: 269

Cyrillus Alexandrinus

comm. in Isaiam 440, 41-55: 365

Demetrius Cydones

ep. 389: 437

Demosthenes

or. XIX 65: 469

or. XXV in Aristog. 32, 8-33, 1: 118

Dio Cassius hist. I 90, 4-9: 118

Diodorus Siculus bibl. hist. XIV 27, 1-5: 133

XVI 31, 4-5: 135

XXXII 20: 120

Dionysius Halicarnassensis ars rhet. I 257, 7-10: 148 257, 10-12: 146

Epanagoge

40, 61: 359

Euripides

Hecuba 558-561: 474

Hippol. 612: 238

Eusebius Caesariensis

Vita Constantini I 6: 118

I 19, 2: 125 
Eustathios Thessalonicensis de emend. Vita mon. §144 (160, 10162, 10): 411 opera minora $10(178,87-180,49)$ :

268

$12(197,64-66): 421$

13 (205, 91-93): 420

13 (207, 86-88): 421

13 (209, 30-33): 420

13 (211, 14-16): 426

13 (221, 47-222, 48): 422

13 (224, 22-24): 422

13 (224, 39): 422

$13(225,30-226,96): 268$

$13(225,45-47): 426$

$13(225,55-56): 424$

14 (242, 33-38): 424

14 (244, 12-15): 423

$14(248,41-46): 411,419$

$16(262,50-52): 421$

$16(264,17-24): 427$

$16(273,22-27): 423$

$16(273,38-41): 426$

$16(279,14-16): 420$

$16(280,49-50): 422$

16 (286, 59-64): 427

comm. in Iliadem (ed. van der Valk)

1, 551, 15-16: 360

3, 17, 374: 238

4, 275, 11-17: 360

comm. ad Homer. Odysseam (ed.

Stallbaum)

II 291, 39-40: 7

Euthymius Malaces

II 48-49: 268

\section{Galenus}

de locis affectis 428, 5-6: 362

Georgius Acropolita

Opera I 188: 437, 445

I 188-189: 444

I 189: 445

Georgius Cyprius

Enc. VIII 324: 435

Enc. in Michaelem VIII (347): 441

VIII (348, 24-351, 22): 444

Georgius Monachus

Chron. 619, 19: 4
Georgius Sphrantzes

Chron. 28, 9-23: 94

124, 16: 98

124, 18: 98

124, 20-21: 98

124, 22: 98

126, 1: 97

126, 4: 98

126, 5: 97

126, 21-128, 6: 99

126, 27: 97

128, 17: 98,99

128, 21: 98

Gregorius Acindynus

ep. 1, 10-19: 283

Gregorius Corinthius

De compositione 321, 203-206: 414

Gregorius Nazianzenus

ep. $46(42,8 f):$.

ep. 51 (47f.): 416

ep. 80 (71): 416

ep. 93 (78): 417

or. 4: 293

or. $8,8: 21$

or. $11(4,15-16): 159$

or. $11(5,3-5): 159$

or. $11(5,14-16): 159$

or. $43(24,43): 33$

Gregorius Nyssenus

de vita Macrinae 1: 19

16: 21

11: 21

Heliodorus

9, $5: 347$

Hermogenes

Id. I 214, 12-21: 109

II 411, 2-7: 113

opera 63-64: 427

69-70: 427

213-413: 425

263-264: 426

268-269: 426

309-311: 427

320-321: 427

330: 426

339: 425

prog. 23, 1-6: 139 
Stat. VII 79, 7-12: 109

Herodotus

I 8, 10-11: 109

VI 31: 67

VI 127: 396

Hesiodus

fr. 286: 230

op. 143-147: 362,375

scutum 175: 234

Hildegard Bingensis

causae et curae $2(162,3 \mathrm{ss}$.): 194

Homerus

Ilias 2, 87-88: 253

2, 89: 254

2, 453: 67

2, 459-463: 247

2, 469-471: 255

3, 23: 236

3, 35: 225

3, 272: 115

4, 350: 251

5, 299: 236

5, 499: 253

5, 518: 363

5, 846: 363

6, 506-507: 242

10, 173-174: 133

11, 67-69: 226

11, 173-176: 236

11, 790-805: 134

14, 348: 228

15, 263-264: 242

15, 504: 67

16, 76-79: 116

16, 583: 247

17, 755-756: 247

18, 56: 224

19, 259: 115

21, 12-14: 255

22, 93-94: 252

23, 597-599: 226

Odyssea 6, 130-132: 236

6, 163: 224

7, 115: 362

11, 589: 362

12, 294: 152

13, 106: 240

19, 163: 227
Horatius

ep. 1, 3, 23s.: 193

Ioannes Cantacuzenus

hist. $3,96.1: 88$

3, 97.4: 88

3, $97.16: 88$

3, $98.2: 88$

or. 43,1-2: 444

Ioannes Chortasmenus

202, 14s.: 416

217, 24: 438

Ioannes Chrysostomus

in Genesim PG 53, 186: 34

ad illuminand. catech. 2 , PG 49, 231:

27

ep. X ad Olymp. 1d: 118

hom. XLIV in I ep. ad Cor., PG 61, 380, 31-32: 118

in epistulam ad Eph. PG 62, 48: 27

in epistulam ad Thess. PG 62, 412:

27

Ioannes Cinnamus

ethopoeia 32-36: 360

epit. rerum hist. VI 13 (290, 14-21):

267

Ioannes Climax

scala paradisi II 7: 50

Ioannes Geometres

progymn. 4, 14: 362

Ioannes Malalas

chrongr. $15: 4$

72: 4

83-84: 10

109: 9

135ss: 10

151-152: 8, 10

233: 8

237: 8

251: 4

252s.: 10

263s.: 9

265: 9

266: 4

327: 4,8

332-333: 8

333-334: 8

352ss: 10 
359: 4

403: 2

406: 7

407-408: 10

408-409: 11

Ioannes Sardensis

208-209: 360

comm. in Aphth. progymn. 217: 461

Ioannes Scylitzes

3, 18: 114

3, 26-4, 35: 107

52, 67-84: 126

261, 10-12: 126

272, 91-273, 36: 130

288, 1-5: 130

308, 10-309, 33: 135

338, 45-339, 63

Ioannes Siceliotes

VI 99, 10-13: 412

414, 20-22: 412

Ioannes Tzetzes

comm. in ranas 897: 270

Ioannes Zonaras

epit. hist. 2, 102, 24-27: 363

XIV 138: 7

523: 130

XVII 7 (553, 4f. 13-17): 215

XVII 7 (555, 9-10): 215

Isidorus Pelusiota

ep. 1412: 287

ep. 1555: 287

ep. 1697: 287

Iulianus

contra Galilaeos fr. 9, 13-17: 287

ep. 42 (73, 1-15): 292

ep. 89 b97-98: 363

or. $1,5 b-10 b$ : 56

Leo Diaconus

hist. 5, 9-14: 109

5, 19-22: 109

$6,15-7,8: 116$

$6,16-17: 117$

$6,16-7,8: 123$

7, 3-7: 117

9, 18: 113

10, 14-15: 113

12, 5-11: 114
12, 5-13, 10: 112

12, 11-13: 117

12, 11-16: 114

12, 16-21: 114

12, 21-13, 3: 114

12, 22: 133

13, 3-10: 114, 134

13, 11: 115

19, 12-20, 7: 116

19, 23-20,1: 117

20, 9-10: 117

20, 10: 117

20, 12-13: 117

20, 13-18: 117

20, 13-21, 13: 116

20, 15: 119

20, 18-24: 117

20, 24-21, 11: 118

21, 12-23: 118

21, 14-16: 118

21, 24: 117

21, 24-22, 1: 119

22, 1: 119

23, 19-20: 120

24, 6-7: 119

24, 3-6: 120

39, 14-41, 21:121

40, 1-3: 135

42, 1: 123

42, 1-7: 121

42, 1-43, 18: 120

42, 2: 121

42, 4: 122

42, 6: 121, 122

42, 7-10: 122

42, 10-21: 122

42, 21-43, 8: 122

43, 8-15: 122

43, 9: 123

43, 15-18: 123

43, 19-20: 124

46, 13-14: 120

48, 19-20: 129

49, 11-50: 125

50, 1-14: 125

50, 14-20: 125

51, 3-4: 126

55, 2-4: 126 
56, 13-14: 126

63, 24-64, 12: 130

66, 3: 126

68, 3-69, 3: 127

69, 3-22: 127

69, $22-70,2: 125$

72, 1-18: 127

$72,23-73,3: 127$

72, 23-74, 12: 127

$73,1: 127$

$73,3: 127$

73, 3-9: 128

73, 9-11: 128

73, 11-18: 128

$73,18-74,6: 128$

74, 6-12: 129

$77,12-15: 130$

81, 13-83, 1: 130

$85,15-86,6: 129$

94, 16: 133

95, 5-19: 129

102, 22-23: 133

107, 11-13: 134

130, 19-23: 131

130, 23-131, 4: 132

130, 19-132, 14: 130

131, 2-4: 132

131, 4-8: 132

131, 8-12: 132

131, 13-15: 133

131, 19-23: 133

131, 23-132, 7: 133

132, 7: 134

132, 7-11: 133

132, 9: 134

132, 9-10: 134

132, 11-14: 134

132, 16: 129

132, 24-134,1: 134

153, 22-154, 18: 135

154, 23-155, 13: 135

158, 14: 129

159, 2-9: 135

172, 14-17: 124

177, 4-5: 137

Leo Grammaticus

chronographia 119, 1: 4
Lexica Segueriana

140, 13: 413

168, 12: 413

172, 7: 413

Libanius

ep. 33: 152,155

763, 3: 65

1259: 278

or. $1,48: 56$

1, 156: 59

1, 171: 63

1, 239: 63

18, 8: 57

18, 10: 62

18, 41: 57

18, 53: 67

18, 57: 67

18, 58: 67

18, 60: 67

18, 61: 67

18, 62: 64

18, 66: 64

18, 68: 63, 64

18, 81: 57

18, 105: 58

18, 106: 58, 67

18, 162: 65

18, 181: 57

18, 184: 66

18, 193: 66

18, 242: 63

18, 243: 63

18, 255: 67

$61(329,2-330,6): 145$

(330, 6-8): 145

(330, 9-332, 11): 145

(331, 11-14): 154

(332, 1-11): 145

$(332,12-335,2): 145$

(333, 10-11): 154

$(334,13): 154$

$(335,2): 151$

$(335,3-8): 146$

(335, 6-8): 154

$(335,7-8): 151$

(335, 9-10): 154

(335, 12-13): 154 
(335, 14-16): 154

(335, 17-18): 155

(336, 4-5): 151

(336, 6-8): 151

(336, 11-12): 154

(336, 16-18): 152

(337, 3-4): 152

$(337,16-338,20): 146$

(338, 1-14): 151

(338, 8-9): 155

(339, 1-11): 146

(339, 11-340, 3): 146

(340, 2-3): 155

(340, 4-13): 146

(340, 14-341, 7): 146

(341, 3-4): 154

(382, 10-13): 150

or. $64: 155$

op. VIII

460-464: 468

502-504: 464

504: 474

504-505: 464, 472

504-507: 464

505: 465

506: 474

508: 472

508-511: 464

510-511: 474

511-516: 464

512: 471

516: 464

516-517: 471

516-518: 464

520-523: 464

524: 472

524-527: 464

526-527: 471

537: 537

537-538: 464

Lucas Notaras

II 196, 1s.: 412

Lucianus

de salt. 58-59: 364

hist. Conscr. 305, 16-306, 2: 109

Tim. 2, 6-8: 126

Phal.. 1,2,6-7: 126
Macrobius

sat. 7,11, 9: 194

Manuel Holobolus

or. I (32, 16-34): 434

I $(45,36): 444$

II (51-77): 432

Manuel Palaeologus

ep. 27, 8-9: 283

ep. 28, 18-19: 284

panegyr. 223-238 (ed. Boissonade): 438

Marinus Neapolitanus vita Procli 28: 7

Mauricius strateg. VIII 1,4: 134 IX 4: 134

Mazaris

10, 12-16: 97

40, 27-56, 10: 97

59: 284

98, 10: 278

98, 7-15: 96

Menander Rhetor

76: 433

$368,1-377,30: 263$

372, 25-28: 54

418, 5- 422, 4: 54

419, 1-15: 54

Michael Attaleiates

historia (Pérez Martin) 212, 11-12: 3

296, 20

Michael Gabras

ep. 49: 439

52: 439

281, 6-10: 439

281, 17-19: 439

284, 3-9: 439

286: 439,446

313: 439

344: 439

358: 279

Michael Glycas

Annales 471: 5

Michael Haplucheir

Dramation 12-123: 280

Michael Psellus

Chronographia (Impellizeri)

I 2: 213,220 
I 3: 216

I 4: 214, 216, 221

I 5-9: 216

I 10-17: 216

I 13-17: 214

I 16: 214

I 18: 214, 216, 221

I 19-22: 216

I 22: $215,220,221$

I 23-26: 217

I 30-33: 217

I 31: 221

I 32: 221

I 32-33: 221

I 34: 217

I 36: 220

II $1: 218,221$

II $2: 218-221$

II 3: 221

II $4: 220$

II 6: 218

II $7: 220,221$

II 8: 220

II 9: 219,221

II 9.7f:: 221

II 10: 219

VI 15, 10-13: 233

VI 199 (II 148): 297

Encomium Xiphilini (ed. Sathas)

438-440: 296

ep. 5 (96-98 ed. Maltese): 297

ep. 44 (ed. Sathas): 415

ep.175 (ed. Sathas): 293

ep ad.Xiphilinum (ed. Criscuolo)

1, 1-10: 294

1, 3: 293

41: 294

84-89: 298

123: 298

138-150: 294

170-172

205-217: 298

214-217: 299

historia syntomos (Aerts)

52, 36-43: 6

106, 43-108, 49: 215

or. pan. $4(56,27): 282$

or. pan. $6(98,261-265): 263$ or. pan. $6(101,341-342): 263$

poemata $7,177-179: 413$

de omnifaria doctrina (Westerink)

80: 361

de operatione daem.125: 410, 411

theologica I 98, 44s.: 412

Manuel Holobolus II 68-75: 441

Maximus Planudes

Basilikos logos

1, 25-27: 437

4: 435

Menander Rhetor

de encomiis 76-78: 381

78-82: 381

$368,8-369,17: 383$

$369,18-371,14: 383$

Nemesius

de natura hominis 1, 450: 374

Nicephorus Basilaces

ep. 3 (114, 4-5): 372

or. A praefatio 9, 5: 367

or. A3 (3, 18-20): 372

or. A3 (3, 24-26): 357

or. A3 (3, 25-28): 372

or. A6 $(4,32-5,5): 372$

or. $\operatorname{A} 12(7,15-8,13): 372$

or. A13 $(9,5): 367$

or. B1, $20(18,19-19,2): 360$

or. B1, 20-21 (18, 19-19, 24): 372

or. B4, 7 (79, 16-18): 372

or. C $(92,6-14): 360$

or. C $(92,12-14): 372$

or. C, Melete 5-6 (96, 4-97, 5): 371

or. C, Melete $8(97,19-21): 371$

or. C, Melete 10 (99, 3-4): 371

or. C, Melete $13(101,1-2): 371$

or. C, Melete 14-15 (101, 3-13): 371

or. C, Melete $18(103,30): 371$

Progymnasmata (Pignani)

23 (49-100): 361

23, 1-2: 374

49, 22-23: 367

51, 5: 366

51, 18: 366

51, 19-20: 366

51, 26-27: 366 
51, 29-31: 366

51, 44-45: 366

51, 48: 366

54, 50-51: 367

54, 75-90: 372

$55,75: 362$

281: 360

Nicephorus Chumnus

II 14: 435

II 52-53: 440

enc. in Andronicum II 1, 12-2,22:

443

Nicephorus Gregoras

ep. 23 (269-273 Leone): 302

ep. 37, 25: 279

Nicephorus Phocas

de velitat. XXII 23-29: 134

XXXIII 3-7: 131

Nicetas Choniates

ep. 13, 4: 229

hist. 3, 49-50: 258

3, 52-54: 258

3, 56-57: 258

4, 70-71: 230

5, 38: 238

5, 92: 231

6, 19-20: 255

7, 15: 238

8, 32: 240

8, 90-91: 248

13, 40-41: 255

14, 56: 239

17, 33: 241

22, 73-74: 250

23, 4-5: 244

26, 73-74: 241

36, 63-64: 241

41, 12-13: 247

42, 25: 247

43, 23: 240

49, 31-32: 245

54, 69: 224

55, 21-22: 230

56, 49-57, 52: 248

57, 51: 248

59, 18-19: 254

61, 81-82: 237, 238

62, 90: 244
63, 16-17: 254

63, 33-35: 237

64, 61-63: 230

65, 80-81: 232

67, 47-48: 233

70, 27-30: 238

70, 29: 234, 239

71, 55-56: 231

71, 56-57: 233

$73,7: 244$

$74,45: 231$

77, 24: 237

86, 68-69: 234

87, 9: 240

$89,56-57: 225$

90, 88-90: 239

90, $94: 236$

90, 94-95: 239

91, 22: 230

95, 26: 224

$95,28: 224$

96, 54: 239

98, 95: 238

103, 2-3: 239

103, 5: 241

104, 44: 256

105, 71: 239

106, 83: 239

111, 43-112, 44: 249

112, 46: 254

113, 82-84: 237, 253

114, 25: 240

114, 30-115, 31: 248

115, 48-49: 236

120, 77-78: 245

123, 76: 235

123, 80-87: 252

127, 68-69: 224

128, 6-7: 232

131, 5-6: 244

133, 67-69: 250

136, 56-58: 249

136, 62-63: 238

136, 56-58: 249

137, 86-87: 233

138, 9-10: 235

138, 23-24: 248

139, 50: 224 
139, 55: 234

140, 77-78: 229

141, 88-89: 242

142, 28: 229

142, 40: 239

143, 46: 231

143, 47-51: 227

143, 51-52: 247

$145,2: 253$

145, 2-3: 247

145, 9-10: 247

146, 39-40: 241

148, 92-94: 249

150, 34: 242

154, 60: 235

151, 60-62: 226

154, 62-63: 235

154, 64: 235

156, 21-22: 252

159, 12-13: 240

159, 13-14: 237

160, 25: 251

169, 5-6: 224

170, 35-171, 36: 225

175, 31-32: 235

182, 33-34: 239

182, 54-56: 252

183, 66: 244

186, 63: 231

187, 16: 240

187, 90-92: 225

188, 36-38: 235

191, 11: 239

193, 63-64: 224

195, 35: 239

201, 94: 224

203, 71-74: 232

205, 30-31: 241

205, 32-33: 231

205, 37-38: 231

206, 50-51: 227

221, 41: 253

222, 63-64: 248

224, 39: 229

225, 48-50: 251

226, 80: 248

227, 23-25: 254

227, 23-228, 30: 240
227, 25-228, 26: 228

228, 40: 255

229, 61-62: 246

230, 79: 253

231, 5-6: 241

232, 29: 248

232, 47-233, 49: 226

233, 51-52: 248

242, 16-19: 250

243, 37: 241

244, 64: 248

248, 68: 254

248, 73-74: 238

248, 74-75: 251

251, 47-50: 252

253, 91: 243

254, 15: 234

254, 15-16: 245

255, 36-37: 246

257, 69: 236

263, 24-25: 231

264, 50-52: 246

264, 52-53: 254

264, 58: 239

266, 24: 241

267, 34: 239

269, 83-84: 241

269, 94-95: 229

270, 25-26: 254

272, 65: 234

273, 1: 247

275, 15-276, 19: 225

276, 34-35: 227

277, 53: 243

278, 77-80: 234

280, 31: 226

282, 80: 255

283, 9-10: 234

283, 15: 242

283, 24: 244

283, 23-24: 243

284, 29: 233

285, 59-60: 244

286, 88: 237

288, 55-57: 236, 239

289, 71-72: 233

289, 74-75: 233

289, 84-89: 233 
291, 53-55: 233

292, 56-57: 226

292, 69-71: 242

296, 74-75: 250

296, 84: 243

296, 86: 239

297, 14: 244

298, 17: 243

298, 25-27: 242

301, 4: 253

301, 4-6: 237

301, 8-9: 247

301, 12: 247

301, 19-20: 250

304, 1-2: 247

304, 88: 234

304, 89-92: 243

305, 28-29: 244

306, 56-57: 242

307, 79-81: 238

308, 7-8: 257

308, 8-10: 246

308, 12-13: 246

309, 38-39: 245

310, 45-47: 228

$311,83: 252$

$312,20-313,1: 237$

317, 11-13: 245

319, 45-51: 256

321, 15-2: 241

321, 21-23: 235

321, 23-24: 246

321, 24: 240

322, 33: 248

322, 35-37: 251

324, 7-9: 249

324, 7-11: 228

324, 10-11: 248

$325,30-31: 255$

334, 86-89: 251

334, 91-92: 247

335, 4-6: 255

338, 82-83: 227

338, 86: 240

338, 89-91: 249

341, 79: 239

341, 79-80: 248

342, 2: 240
342, 82-86: 242

342, 83-84: 247

343, 35: 241

343, 40: 239

348, 65-66: 234

348, 88-349, 89: 254

349, 11-12: 226

$349,18-19: 255$

$352,9: 254$

356, 27-28: 246

$358,87: 237$

$362,1: 249$

362, 81-84: 237

362, 82: 238

364, 31-32: 234

367, 18: 254

372, 57-373, 59: 240

377, 53-54: 242

377, 54: 241

385, 57-59: 245

389, 73: 225

392, 6-66: 247

392, 67: 239

392, 69-70: 250

397, 95: 245

398, 15-16: 234

407, 82-85: 243

410, 69-70: 241

415, 4-5: 232

415, 13-14: 236

425, 64: 244

427, 31-32: 228

429, 70-71: 243

429, 71: 240

430, 14: 239

430, 16: 239

432, 64: 240

433, 79-81: 255

434, 19: 254

435, 39-42: 238

437, 16-23: 241

437, 20-21: 241

439, 72-73: 244, 248

440, 81-82: 254

440, 82-83: 244

441, 15: 248

442, 32: 248

445, 43-45: 228 
446, 51-53: 227

447, 94: 229

447, 97: 229

448, 19-21: 253

449, 44-45: 242

451, 73-74: 254

456, 80: 240

456, 87-88: 243

457, 93: 241, 243

457, 93-94: 241

460, 74-75: 228

463, 79-80: 226

464, 8: 250

464, 9-10: 234, 251

464, 9-12: 251

468, 11: 239

468, 13: 236

471, 2-3: 255

473, 56: 225

473, 58-59: 240

477, 79-81: 227

477, 90: 227

477, 92-93: 248

484, 91-485, 3: 257

484, 92-485, 93: 226

485, 5-6: 255

486, 50: 240

488, 91-94: 243

489, 40: 239

489, 41-42: 235

490, 64-65: 226

490, 82-83: 224

490, 85-86: 224

491, 7-8: 254

491, 12-14: 254

492, 43-48: 257

492, 46: 250

492, 47: 255

493, 80-81: 226

507, 48: 253

510, 41-42: 230

511, 70: 239

511, 73: 247

511, 73-512, 75: 241

513, 10: 239

513, 11: 239

514, 44: 232

521, 94-95: 253
522, 30: 240

523, 38-39: 228

524, 76: 238

524, 76-77: 238

524, 79-81: 239

$525,8-9: 244$

527, 50: 245

527, 53-54: 245

527, 67-68: 240

528, 74-75: 240

534, 71: 226

537, 56: 231

540, 39: 227

548, 3-5: 232

548, 6-7: 232

558, 30: 252

558, 32: 225

558, 44-45: 240

559, 62: 224

561, 22: 237

566, 21-22: 230

566, 41: 248

566, 42: 239

569, 8-10: 241

578, 47-48: 246

578, 51-53: 246

578, 56-57: 246

579, 59-65: 236

579, 65-69: 236

581, 29-30: 233

585, 45: 254

589, 46-47: 253

589, 62-63: 234

590, 66: 238

590, 87-88: 242

592, 33: 227

592, 35: 249

593, 51: 230

593, 52-53: 230

593, 56: 240

593, 66-67: 248

600, 54: 244

604, 37-40: 252

606, 5: 240

606, 6-7: 254

607, 22: 238

607, 22-23: 250

610, 1-5: 245 
611, 28: 252

613, 62: 229

620, 78-80: 254

621, 8-9: 239

623, 77-79: 231

625, 42-44: 245

625, 44: 228

627, 78-79: 239

628, 13: 254

628, 15-16: 253

630, 79-81: 232

631, 14-16: 239

636, 64-65: 231

642, 83: 253

648, 52-54: 229

652, 66: 255

652, 69-70: 225

654, 43: 253

or. $13: 267$

or. 17: 267

Nicolaus Lampenus

II 31: 435

Nicolaus Myrensis

progymn. 69: 467, 471

Nonnus

Dionysiaca 2, 223: 362

3, 171-172: 362

Opsarologus

1-10: 94

Ovidius

ars 1, 358: 193, 194

ep. 2, 2, 9s.: 193

ep. 2, 5, 57: 193

Ibis 435: 193

metamorph. 10, 298-502: 368

Philostorgius

hist. eccl. VIII $11(112,1)$ : 410

Philostratus

imagines 1.9.6: 362

Philotheus

kletorologion 195: 264

214-219: 270

217: 270

Photius

bibliotheca 190: 359

228: 4 246b: 4

V 228 (246 b5): 411

contra Manichaeos PG 102, 73: 277

ep. 2, 154: 4

Plato

leges $729 \mathrm{c}: 363$

845e: 396

881d: 363

Phaidr. 253e: 133

Politeia 509b: 347

Res publ.377E-378E: 291

390AB: 291

395B: 291

396CD: 291

Tim. 90a: 374

Polybius

hist. III 63, 10-12: 118

XXXVI 14, 2: 120

Porikologus

A 1-11: 94

A 20: 95

A 27-31: 94

A 44: 95

A 58: 95

A 86: 95

A 114: 95

A177-181: 95

Procopius

bella II 14,5: 204

de aedif. 1,2,5-12: 471

Procopius Gazensis

comm. In Isaiam 2137, 47-54: 365

Pseudo-Apollodorus

Bibliotheca (ed. Wagner) 3, 181-186:

364

Pseudo-Codinus

de off. 132, 12: 86

133, 7-137, 4: 75

134, 3-9: 92

134, 9: 88

134, 12-135, 5: 92

135, 8: 88

136, 7-12: 80

138, 16: 95

138, 21: 93

139, 2: 95

139, 3: 95

139, 10: 86 
141, 1-166, 32: 92

143, 2-6: 89

141, 3: 87

143, 2-145, 11: 89

143, 7-144, 5: 89

144, 6-8: 90

144, 8: 86

144, 8-145, 1: 90

145, 1-6: 90

145, 4-9: 90

145, 9-13: 90

145, 10: 87

147, 17-148, 3: 71

148, 2: 92

149, 15-151, 10: 98

150, 7-151, 10: 98

150, 10-12: 98

150, 11: 87

153, 3-6: 90

155, 4: 86

155, 19-20: 90

164, 4: 86

169, 22: 86

172, 9: 86

175, 13: 86

181, 27: 86

182, 15-16: 95

187, 3: 86

188, 20-21: 95

190, 19-21: 77

191, 1: 86

204, 24-207,10: 76

207, 20: 88

207, 20-21: 78

207, 29: 88

208, 14-17: 88

209, 4: 88

209, 14: 89

210, 23: 86

212, 6-14: 78

212, 8: 88

213, 5-9: 77

215, 4-5: 86

215, 11-215, 29: 79

216, 5-217, 20: 78

218, 21-28: 78

219, 22-26: 77

219, 63-66: 93
$235,11: 86$

249, 16: 86

250, 14: 86

250, 21-251, 24: 81

274, 24-25: 88

280, 2-7: 88

284, 3-16: 71

308, 18-19: 95

320, 12-13: 76

Pseudo-Lucianus

Erotes 23: 366

Pseudo-Lycophron

Alexandra 828-830

Pseudo-Quintilianus

decl. maior 4, 17: 200

5, 13: 200

13, 1: 197

13, 2: 198

13, 3: 198

13, 4: 198

13, 5: 199

13, 7: 197, 199

13, 9: 198

13, 10: 199

13, 13: 198

13, 14: 199

13, 16: 197

decl. min. 244, 6: 200

Pulologus

188-193: 92

271-281: 93

309-317: 94

Quintilianus

Inst. II 4, 3: 468

IV 1, 7: 200

V 13, 21: 200

VI 1, 32: 467

VIII 3, 62: 466

VIII 3, 66: 469

VIII 3, 67-68: 466

Rhetores Graeci

VIII 411, nr. 52: 133

Rhetorica ad Herennium

III 20,23: 473

IV 39.51: 469 
Romanus Melodus

Cant. 40, 1. 4-5: 117

Scholia in Lycophronem

829 (ed. Scheer): 364

Scipio Carteromachus

PG 35, 356C: 413

Seneca Pater

Contr. 2, 6, 7: 196

7, 7, 1: 194

Seneca

Phoen. 448: 193

Sophocles

El. 25: 242

Statius

Theb. 1, 77: 194

Stesichorus

fr. 10: 229

Suidae Lexicon

I 541: 414

II 162, 4: 279

Symeon Magister

ep. 89, 23-32: 236

Synesius

Dion 4, 31-32: 367

ep. 101 (169,7s.): 278

ep. $105(188,17-189,1): 367$

Theodorus Ducas Lascaris

ep. 172, 12-15: 411

Theodorus Metochites

misc. 1, $1(20,1)$ (Hult): 301

1, 1(20, 2-4): 301

9, 1,5 (90, 3-4): 301

Theodorus Prodromus

carm. hist. 18, 71: 362

Theodorus Studites

ep. 1: 22

ep. 3: 157

ep. $4: 27$

ep. $77: 20$

ep. 395: 23

laudatio Platonis 808A-B: 16

magna catechesis 29: 359

208, 2-15

Theon Alexandrinus

prog. 119, 14-21: 137
Theophanes Continuatus chron. 63, 22ss.: 415

Theophylactus Achridensis ep. 48 (295): 417

PG 126, 309: 279

Thucydides

historia I 49: 67

II 41, 4: 347

II 70: 322

VII 67, 4: 132

VII 8, 1-3: 133

Vergilius

Aen. 6, 287: 194

Georg. 4, 220-222: 197

Vita Eustratii

1, 367, 1-368, 2: 385

1, 368, 9-12: 405

Vita Euthymii Iunioris 4 (168): 378, 381

Vita Euthymii Sard. 1, 21, 1-3: 393

Vita Evaristi

1, 295, 2-296, 2: 385

1, 296, 2-10: 383

1, 296, 24-28: 389

2, 296,30-32: 389

Vita Georgii Amastrensis 1,4-2,3 (§1): 387, 396

2, 1s.: 405

3 (2, 9-12): 392

Vita Gregorii Decapolitae 57-60: 399, 406

Vita Ignatii

PG 105, 489AB: 403

489B: 403

Vita Maximi Confessoris

68A: 385

Vita Methodii

1244D: 404

1245B-C: 388

Vita Michaelis Syncelli

44, 4-9: 385

Vita Nicephori patriarchae 139, 11-140,5: 387

139, 12s.: 394

139, 13-140, 2: 394 
139, 20s.: 386

140,1: 393

Vita Nicetae Medic.

1, XVIII: 390

1, XIX: 394

3, XIX: 399

Vita Nicolae Studitae

PG 105, 863B: 384

Vita Petri Atroënsis

65 (1, 1s.): 396

$65(1,3-6): 397$

$65(1,4): 395$

$67(1,35-37): 397$

$67(1,37): 397$

Vita Platonis

2, 804C: 393,399

Vita Stephani iunioris

3, 91, 5s.: 390

93, 12-14: 399
Vita Tarasii

$\S 1,1-13$ (69): 388

$\S 1-3$ (69-71): 380

§ 3, 3s. (71): 388

Vita Theodori Studitae

233A: 385

233B: 407

Vita Theophylacti Nicomed.

1, 71: 393

Xenophon

Anabasis

III 4, 4-5: 118

VI 5, 14: 133

Zenobius

1, 93: 236

2, 79: 244

2, 93: 244 


\section{c) Index nominum et rerum}

Ablakka 8

Abraham 20, 114

Abydos 214, 215

Achilleis 89

Achilles 89, 90

Adler 89, 241, 246, 247

Adonis 359, 361, 364-367

Adrianopel 72, 131

Adrianos 48

Ägypten 136, 152, 229

Aelianus 470

Aelios Aristeides 141, 142, 144-153, 414

Aesopus 100

Affe 233

Agatharchides 336

Agathias 106, 136, 139

Aischylos 336

Ajax 464, 465, 471

Akathistoshymnos 422

Akindynos $\rightarrow$ Gregorios

Akro-Korinth 252

Akropolites $\rightarrow$ Georgios

Albeneka, protospatharea 23

Alemannen 57

Aleppo 215

Alexander 269, 272, 291, 464

Alexios I. 101

Alexios II. Komnenos 224, 240, 247

Alexios III. Angelos 224-228, 232, 243, 255

Alexios V. Dukas Murtzuphlos 239, 248

Alexios Apokaukos 90, 91, 93

Alexios Axuch 247, 253

Alexios Branas 225, 237, 242

Alexios Ibanko 240, 241

Alexios Komnenos (Sohn des protobestiarios Ioannes) 250

Alexios Komnenos, protosebastos 226, 248, 249
Alexios Porphyrogennetos 427

Ali Sa'if-ad Daulah 117

Alkibiades 321, 322

Allegorische Auslegung 287

Alliteration 392, 394, 395, 419, 421, 428

Altes Testament 257

Amalthea 257

Amantios kubikularios 11

Ameise 253

Ammianus Marcellinus 151

Amphilochios von Ikonion 179

Anaphora 419, 420, 428

Anastasius I. 1, 5, 6, 10, 11

Anaximenes 109

Andreas Darmarios 102

Andreas von Jerusalem 182

andreia 373, 374

Andronikos I. Komnenos 224-229, 232, 234, 236, 237, 239-244, 246, 251, 254, 255

Andronikos II. 70-72, 79, 80, 99, 100, 102, 435, 439-441, 444

Andronikos III. Palaiologos 69, 74, 75, 78-80, 82, 84, 87, 99, 102

Andronikos Lapardas 234, 243

Angeloi 263, 274

Angelos $\rightarrow$ Konstantinos

Anna, Nonne 15

Anna Komnene 86-88, 101, 108, 138, 225, 240

Anna Palaiologina 80

Anna von Savoyen 444

annominatio 392, 393

Anonymität 108

Anrede 66, 98, 391, 437, 444

Anrede (Stadt) 151, 157

Anthologia Graeca 17, 335

Antikerezeption 110

Antiochia 8, 59, 62, 65, 66, 125, 127, 129 
Antonios, hl. 23

Antonios, Metropolit von Thessalonike 166

Antonios Kauleas 262, 263

Apfel 229

Aphrodite 359, 361-364, 367

Aphthonios 359, 433, 463, 466

Apis 152

Apollo 8, 362, 374

Apollonius von Tyana 9

Apostoles $\rightarrow$ Michael

Apostrophe 61, 194

Applaus 282, 284

Apros 239

Araber 112-114, 116

Archimedes 6, 7, 336

Architext 143

Areopagites $\rightarrow$ Dionysios

Ares 359, 361-368

Aretaios 336

Arethas 259, 260, 262-275, 279, 416

Argos 257

Arianischer Streit 288

Aristainetos 151

Aristeides $\rightarrow$ Aelios

Aristokratie 14, 18, 50, 102, 357

Aristophanes 144, 340

Aristophon 328

Aristoteles 109, 271, 296, 340, 434

Armer 190, 191, 193, 196-198

Armenier 241

Arnold von Harff 206

Arrhythmia 427

Arrian 346

Arslan $\rightarrow$ Kilij

Asan $\rightarrow$ Isaakios

Ascheregen 127

Askese 60, 404

Asklepion 4

astikos 298

Astrologe 8

Astrologie 8, 296

Astronomie 296, 300

Asyndeton 158, 308
Athanasia von Aigina 18

Athanatoi 134

Athen 2, 3, 57, 250, 409, 415

Athene 224, 225, 464

Athos Iberon 426: 160

Athyras 232

Atreus 115

Attaleiates $\rightarrow$ Michael

Attika 7

Attisches Griechisch 87, 340, 342

Auditorium s. Hörer, Hörerschaft, Zuhörer

Augustat 59, 62

Augustus 56-58

Augustinus 53

Ausruf 154

Autobiographie 23, 53

Autohagiographie 23

Automatische Klassifikation / Klassifizierung 308-310, 312, 319, 320, 323$327,333,337,338,341,342,347$

Autor 368

Awaren 422

Axuch $\rightarrow$ Alexios

Babylon 149

Bad 215

Bär 241

Bagoas 360, 371, 372

Baiophoros $\rightarrow$ Georgios

Bankett 228, 259, 261, 262, 269-273

Barbaren 122, 235

Bardanes $\rightarrow$ Georgios

Bardas Phokas 214, 216-218

Bardas Skleros 214-218

Bari 126

Basilakes $\rightarrow$ Nikephoros

Basilakios 253

Basileios I. 269, 270

Basileios II. 211-222

Basileios der Große 5, 9, 100, 176, 180, 182, 287, 290-292, 409, 414, 415, 417

Basileios Nothos 123

Basileios parakoimomenos 214-218 
basilikos $\log$ os $54,432-435$

Basilius Minimus 409

Beifall 117 s.a. Applaus

Belisar 118, 119, 123

Bellerophon 10

Belshazzar 8, 10

Berg 298

Bergbau 203

Bernardus Silvestris 192

Bescheidenheitstopos 385-387, 390, 399, 404, 406

Bibel 288, 289

Biene 189-191, 193, 197-199, 253, 254

Bienenlob 200

Bienenmord 200

Binnenreim 196 s.a. Endreim, Reim

Bithynien 20

Bithynischer Olymp 296, 298

Blachen 240

Blachernenkirche 83

Blemmydes $\rightarrow$ Nikephoros

Boaz 21

Boradiotes $\rightarrow$ Theodosios

Boskytion 45

Bosporus 2

Branas $\rightarrow$ Alexios

Brief 13, 14, 16, 23, 24, 176, 230, 232, 238, 241, 293, 294, 295, 297, 298, $391,415,416,446$

Briefdiplomatie 59

Bringas $\rightarrow$ Ioseph

Bronze 473

Bryennios $\rightarrow$ Manuel

Buchstabenverteilung 308, 310, 324-326, $328,333,337-340,347$

Bulgaren 131, 132

Burtzes $\rightarrow$ Michael

C 14-Untersuchung 204

Caesarius 20

captatio benevolentiae 145, 147

Carteromachus $\rightarrow$ Scipio

Cassiodorus 204

Cassius $\rightarrow$ Dio
Cato 301

Chalkedon 410

Chalkutzes $\rightarrow$ Niketas

Chandax 113, 116

Chele 234

Chi-Quadrat-Anpassungstest 312

Chi-Quadrat-Test 307, 318, 321, 323, 324

Chi-Quadrat-Wert 311, 312

Chiasmus 154, 395, 394, 423

Chimaira 256, 464

Chnodomar 64

Choirosphaktes $\rightarrow$ Leon

Choniates $\rightarrow$ Michael, Niketas

Chora-Kloster 76

Chortasmenos $\rightarrow$ Ioannes

Chorus 279

Christen 61, 287

Christentum 287, 288

Chronistik 106, 108

Chrysokokkes $\rightarrow$ Georgios

Chrysostomus $\rightarrow$ Ioannes

Chrysotriklinon 270, 273

Chumnos $\rightarrow$ Nikephoros

Cicero 335, 469

Claudianus 54

Computerprogramm 306

Constantius II. 56, 57, 59, 61, 65

Cordoba 137

Cornutus 336

Crassus 61

Damaskenos $\rightarrow$ Ioannes

Daniel 8

Dante 373

Daphne 360, 362, 374

Daphne (bei Antiocheia) 8, 142

Dareios 8, 291

Darmarios $\rightarrow$ Andreas

David 400

David Komnenos 242

declamatio 196

Dedicatio 379, 380

Dekapolites $\rightarrow$ Gregorios

Deklamation 336 
Deklamatorik 197

Delphin 95, 245

Demetrios, hl. 157, 159-161, 165, 262

Demetrios Kydones 95, 431, 438, 440, 442, 447

Demosthenes 153, 300, 332, 336, 342, $346,347,412-414,469,471,473$

Dendrogramm 307, 312, 319, 320, 325 , $338,339,348,349$

Dernschwam, Hans 207

Desa 238, 249

Despotes 89

Deutsche 227, 232

Diadem 66

Diakonos $\rightarrow$ Ignatios, Leon

Dialog 10, 142, 148

Digenis Akritas 374

Dio Cassius 114

Diocletianus 148

Diodorus Siculus 114

Diogenes von Sinope 336

Dionysios Areiopagites 4

Dionysios von Halikarnassos 335

Dokeianos $\rightarrow$ Ioannes

Donau 131, 240

Donner (Motiv) 411

Doppelspondeus 425

Dorostolon / Silistra 132, 135

Dositheos 255

Doxapatres $\rightarrow$ Ioannes

Drama 10, 280, 362, 363, 367, 368, 372, 430, 464, 467

Drohbrief 127

Dukas $\rightarrow$ Ioannes, Isaakios, Konstantinos

Dyrrhachion 229

Eber 240

Eden 249, 424

Edessa 127

Eid 123

eidos 429, 434, 435

Einzeldeklamation 312

Eirene 15, 17

Ekphrasis 151, 463-472
Elephant 244

Eleusis 142

Elias 270-274

Elision 315

Ellipse 154

Emir von Tunis 127

Emmanuel Rhaul 279

enkomiastikos 434, 435

Endreim 196 s.a. Binnenreim, Reim

Enkomion 54, 138, 144, 149, 158, 160, $165,167,432,434,436,437,440$, 443,447

Ephippos 336

Epidamnos 245

Epideiktik 53, 429

Epigramm 411

Epilog 144, 153

Epiphanie 259-263, 272, 274, 426

Epitaphios 53-60, 62, 67

Epithalamium 288

Epos 67

Erdbeben 141, 148

Eros 357-359, 361-363, 365-375

Erotik 358

Erzähltechnik 62

Erziehungsproblem 292

Erwartungswert 311

Esel 242

Eteokles 464, 472

Ethik 290, 444

ethopoiia 359 , 360, 365-368, 370, 371, 375

ethos 84, 464

Euarestos 389

Eudokia (Athenais) 10

Eugenios Giannules 415

Eunuch 71, 72, 120, 122-124, 126, 133, 137, 221, 271

Euphrosyne Kastamonitissa 235, 239, 241, 243

Euripides 333,335, 340, 346, 474

Eusebios von Kaisareia 107

Eustathios von Thessalonike 7, 238, 257, 280, 411, 419-423, 425, 428 
Eustratios 406

Euthymios Malakes 268

Euthymios von Sardeis 381, 393

Euthymios Zigabenos 374

Eva 424

Familie 18

Familienkult 18

Feldherrnmantel 121

Feldherrenrede s. logos parakletikos

Feldlager 124, 126

Ferdinand I. 207

Festmahl 390

figura etymologica 395

Fischer 150, 151

Fliege 255

Floh 254

Flötenspieler 145, 147

Folter 465

Forum Tauri 229

Fotomodell 59

Franken 236, 237

Freund 20, 60, 63, 73, 230, 278, 295, 318, $361,414,417,439,446$

Freundschaft 238, 297, 415

Friedrich Barbarossa 241

Frosch 253

Fuchs 238

Funktionswort 308, 310, 312, 317, 320

Gabalas $\rightarrow$ Manuel

Gabras $\rightarrow$ Michael

Gallien 57, 59-61, 64

Gallus 57

Garten 229, 230

Gaußsche Glockenkurve 307

Gazes $\rightarrow$ Theodoros

Geburt 378, 381

geistige Nahrung 389

Gemistos Plethon $\rightarrow$ Georgios

Genealogie 374

genos demegorikon 109

Genre 433

Genuesen 444
Georgios Akropolites 437, 442, 444, 445

Georgios von Amastris 387, 392, 396, 398, 405, 406

Georgios Baiophoros 100

Georgios Bardanes 410

Georgios Chrysokokkes 101

Georgios Gemistos Plethon 101, 302

Georgios Kyprios 435, 440, 442, 447

Georgios Monachos 5

Georgios Muzalon 411

Georgios Pachymeres 87

Georgios Pisides 414

Georgios Scholarios 101

Gerichtsrede 196

Germanen 59

Germanenkriege 56

Geschenk 77, 93

Geschichtsschreibung s. Historiographie

Gestik 281, 282, 372

Geza II. 224

Giannules $\rightarrow$ Eugenios

Gift 199

Glaubensbekenntnis 397

Glykas $\rightarrow$ Michael

Götterkritik 291

Gold 203, 204, 206

Gongyles $\rightarrow$ Konstantinos

Gorgonia 19-21, 414, 415

Gottähnlichkeit 197

Grabrede 414, 446

Gras 232

Gregoras $\rightarrow$ Nikephoros

Gregorios Akindynos 283

Gregorios Dekapolites 399, 405

Gregorios von Nazianz 19-21, 101, 158, 176, 181, 294, 359, 409-417

Gregorios von Nyssa 19, 21, 158, 176180, 294, 416

Gregorios Pardos 414, 416

Gregorius I. 5

Griechenland 464

„Griechisches Feuer“ 2, 4, 7, 11

Gyllipos 132

Gymnopaidiai 220 
Hades 362

Hadesfahrt 95

Hafen 146, 151, 206

Hagia Sophia (Konstantinopel) 101, 102, $259,261,262,265-267,471$

Hagia Sophia (Thessalonike) 157

Hagiochristophorites $\rightarrow$ Stephanos

Hagiographie 15, 17, 19, 377

Hagios Lazaros (Konstantinopel) 259

Hamdāniden 117, 119

Handbuch 360, 466

Handschrift 378, 379

Haplucheir $\rightarrow$ Michael

Heidentum 291

Heiligenvita, -viten 289, 377, 403, 405, 407

Heiligkeit 25

Heimat 378, 381, 382, 387

Heinrich von Flandern 239

Heirat 20-23

Hekuba 472

Helena, Kaiserin 83

Helena 253

Helena Kantakuzene Palaiologina 437

Helios 146, 152

Hera 464

Herakleios 211

Herakles 251, 464

Hermogenes von Tarsos 104, 109, 111, $139,421,425-427,433,435$

Herodotos 149, 231, 336, 396

Herrschertugend 62

Hesiod 230, 291

Hesychasmus 289

Heterosexualität 357

Hiat 421

Hiatrate 307, 309, 314, 315, 327, 329, 330, $333,337,338,341,343,347,348$, 350,353

Hiatvermeidung 315, 338, 343, 346, 347, 353

Hieronymus 191

Hildebert von Lavardin 192

Himerios 142, 467
Hiob 400

Hippodrom 120, 211, 252, 271, 273, 277

Hippokrates 335, 346, 450,

Historiographie 8, 105, 107, 108, 212, 415

Historizität 402

Hochzeit 15, 18

Hörer, Hörerschaft 108, 164 s. a.

Auditorium, Zuhörer

Hofdichtung 429

Hofetikette 66

Hofkleidung 92

Hofzeremoniell 436, 441

Holobolos $\rightarrow$ Manuel

Homer 138, 144, 147, 236, 255, 257, 290 292, 293, 413

Homer-Philologie 287

Homiletik 260

Homilie 262, 266, 271, 272

Homoioptoton 392, 395

Homoioteleuton 392, 395, 419-421, 428

Homosexualität 358

Honig 191, 254

Horaia Pege 296

Hut 91, 93

Hyakinthos 362

Hydra 251

Hymnographos $\rightarrow$ Ioseph

Hymnologie 428

Hypatia 4

Hyperbaton 392, 394

Hyperbel 322

Hypertext 142, 143

Hypotext 143, 144

Hyrtakenos $\rightarrow$ Theodoros

Iamblichos 9, 300

Ibanko $\rightarrow$ Alexios

Ibn Yahya 215

Ich 153, 366

Idealbild 222

Ignatios, Patriarch 403

Ignatios Diakonos 380, 387, 388, 405

Ikonenverehrung 381, 406

Ikonoklasmus 13, 16, 165, 166, 404 
Indien 203

Insekten 253

Intertextualität 143

Intitulatio 379,380

Inzest 359, 364-356, 368-370, 373, 375

Ioannes (Evangelist) 410

Ioannes I.Tzimiskes 120, 121, 130-136, 213,216

Ioannes II. Komnenos 247, 248, 250

Ioannes V. Palaiologos 85, 87, 95, 437, 438

Ioannes VIII. 447

Ioannes XIV Kalekas 84, 437, 444, 447

Ioannes Chortasmenos 416, 431, 435, 438, 444,447

Ioannes Chrysostomos 14, 182, 417

Ioannes Damaskenos 178

Ioannes Doxapatres 328

Ioannes Dokeianos 440, 447

Ioannes Dukas 421

Ioannes Italos 295

Ioannes Kamateros 232, 238, 240, 247 , 249

Ioannes Kantakuzenos 70, 72, 73, 76, 78, $79,81,84,85,87,90,91,94,98,102$

Ioannes Lagos 244

Ioannes Malalas 1, 3-10

Ioannes Mauropus 295

Ioannes monachos 181

Ioannes Moschos 5

Ioannes Palaiologos 76, 79

Ioannes von Putze 230

Ioannes von Sardes 360

Ioannes Sardianos 466

Ioannes Sikeliotes 412

Ioannes Skylitzes 219

Ioannes Spyridonakis 226

Ioannes von Thessalonike 158

Ioannes Tzetzes 278, 364, 412

Ioannes Xiphilinos 293-296, 298-300, 415

Ioannes Zacharias 301

Ioannes Zonaras 6, 107, 212

Ioseph Bringas 120, 122-123
Ioseph Hymnographos 406

Ioseph, oikonomos 58

Ioseph Philosophos 440

Ioseph Rhakendytes 300, 434

Ioseph Studites 157-159, 160, 166, 167

Iovianus 61

Isaakios II. 227, 239, 242, 244, 252, 253 , 255

Isaakios Asan 76

Isaakios Dukas 226

Isaakios Dukas Komnenos 250, 251

Isaakios Komnenos 245

Isidoros von Kiev 100, 440, 446, 447

Isokrates 100, 335, 342, 346, 467

Isthmos 245

Italikos $\rightarrow$ Michael

Italos $\rightarrow$ Michael

Iulianus Apostata 5, 8, 53-55, 57, 58, 60 64, 287, 290, 292, 359, 417

Thronerhebung 56, 58

Iulianus Chaldaeus 9

Iulius Constantius 57

Iustinianos I. 101, 119, 130, 269, 270, 273, 410,473

Iustinus I. 130

Jagd 215, 220

Java 205

Kabasilas $\rightarrow$ Nikolaos

Kaıڤós 433

kaisar 74, 78

Kaisareia

Kaisarios 414, 416

Kaiser 108,114, 444, 448

Kaiseranrede 444

Kaiserkritik 123, 127

Kaiserpalast 440

Kaiserrede 431-433, 435-437, 439, 440, 447

Kalamanos $\rightarrow$ Konstantinos

Kalekas $\rightarrow$ Ioannes

Kallikles $\rightarrow$ Nikolaos

Kallistratos 474 
Kalokyres 130

Kalokyros $\rightarrow$ Patrikios

Kalomodios 238

kalydonischer Eber 240

Kamateros $\rightarrow$ Ioannes

Kamel 244

Kannibalismus 322

Kantakuzene $\rightarrow$ Helena

Kantakuzenina $\rightarrow$ Maria

Kantakuzenos $\rightarrow$ Ioannes, Matthaios

Kappadokien 263

Karbonopsina $\rightarrow$ Zoe

Kardinaltugend 382, 434

Kastamonitissa $\rightarrow$ Euphrosyne

Kastrophylax 95

Kathara 47

kathisma 271, 273

Kauleas $\rightarrow$ Antonios

Kephalos 328

Kiev 130, 218

Kilij Arslan 235, 251

Kinyras 359

Klage 54

Klassifikation s. automatische Klassifikation

Klausel, Satzklausel 315, 338, 343, 346, 354

Klauselrhythmik 315, 316

Kleidung 74, 227

Kleinasien 116-119, 207, 439

Kleisura 131

Kletorologion 273

Klimax 57, 118

Kloster 15, 16, 100, 296-299, 411

Köln 206, 207

Körper 151

Kommemoration 20

Komnene $\rightarrow$ Anna

Komnenen 263, 269, 274

Komnenos $\rightarrow$ Alexios, Andronikos I., David, Ioannes II., Isaakios

Konrad III. 224, 237, 238

Konrad, Sohn Wilhelms v. Montferrat 224
Konstantinopel 1, 2, 3, 4, 7, 10, 20, 56, 57, $60,64,69,76,83,85,91,100,124$ 126, 135, 158, 203, 206, 207, 209, $217,224,229,242,246,247,256$, 259, 261, 266, 269, 274, 295, 297, $373,404,426,438-442$

Konstantinos I. 57

Konstantinos III. 5

Konstantinos IV. 132

Konstantinos V. 16

Konstantinos VI. 16

Konstantinos VII. 107, 270

Konstantinos VIII. 211-215, 218-222

Konstantinos IX. Monomachos 263, 295 , 297

Konstantinos Angelos 224

Konstantinos Angelos Dukas 237

Konstantinos Dukas 224

Kontantinos Gongyles 116, 117, 123

Konstantinos Kalamanos 229

Konstantinos Manasses 362, 375

Konstantinos Mesopotamites 224, 226, 244, 246, 250, 254, 255, 257

Konstantinos Palaiologos 79

Kontingenztafel 311

Kontostephanos $\rightarrow$ Stephanos

Konzil von Chalkedon (a. 451) 397

Konzil von Nikaia (a. 325) 397

Konzil von Nikaia (a. 787) 16, 381

Kopist 378, 394

Korydaleus $\rightarrow$ Theophilos

Kosmas Melodos 178, 182

Krankheit 438

Krasis 315

Kreta 112, 113, 123

Krönung 80, 82-84, 213, 234, 437, 441

Kronos 362

Ktesiphon 61

Kumanen 232, 236, 239, 245, 250, 253, 254

Kuropalates 217

Kydones $\rightarrow$ Demetrios

Kyprios $\rightarrow$ Georgios 
Kyrillos von Jerusalem 288

Lagos $\rightarrow$ Ioannes

Lampenos $\rightarrow$ Nikolaos

Laodikeia 66

Lapardas $\rightarrow$ Andronikos

Laskaris $\rightarrow$ Matthaios, Theodoros

Lateiner 234, 237, 242, 244, 246, 247 , $250,256,257$

Laurea $\rightarrow$ Tullius

Lazaros 259, 268

Lehrer 441, 468

Lemnos 152

Leon III. 5, 165

Leon V. 158, 165, 166

Leon VI. 120, 121, 123-125, 134, 160, 259, 260-263, 266-274

Leon Choirosphaktes 261, 274, 275, 279

Leon Diakonos 105, 106, 108, 112, 113 , 115-117, 119-121, 123, 125-127, $130,131,133-138$

Leon Monasteriotes 237

Leon Parakoimomenos 120

Leon Phokas 116, 118, 119

Leon Sguros 238, 250, 252

Leopard 238

Lesen 106, 121, 137, 152, 220, 222, 282

Leser 395

Leserschaft 106

Lexica Segueriana 413

Libanios 4, 53-63, 65, 66, 141, 142, 144, $145,147,148,150-155,227,278$, $305,306,308-310,312,314,315$, $319,320,322,327,328,330-338$, $340,342-347,352,353,413,416$

Literaturtheorie 143

Liutprand von Cremona 108, 115, 125 , 135

Löwe 236-238, 256

logos parakletikos 105, 109, 110, 112, $113,115,117,121,123,124,126$, $130,134,135,136,138$

logos paramythetikos 112

logos presbeutikos 112

logos stratiotikos 130 logos symbuleutikos 112, 134

logothetes 95

Luftverschmutzung 205

Lukas Notaras 412

Lukianos 53, 96, 335, 340, 342, 344

Luxus 221

Madagaskar 206

Magistros $\rightarrow$ Symeon, Thomas

Magnaura-Palast 264, 265, 266, 267

Maiandros 238, 419, 423, 424

Maistor ton rhetoron 260, 263

Makrina 19, 21

Malakes $\rightarrow$ Euthymios

Malalas $\rightarrow$ Ioannes

Mamalos 252

Manasses $\rightarrow$ Konstantinos

Mantzikert 211

Manuel I. 224, 225, 226, 230, 248, 252, 254

Manuel II. Palaiologos 97, 99, 283, 431, 435, 437, 438, 442, 446

Manuel Bryennios 438

Manuel Gabalas 437, 438, 440, 442, 446, 447

Manuel Holobolos 97, 434, 435, 437, 438, 441

Maozamalcha 60-63

Marcianus graecus 608: 101

Margaret Maria 231

Maria, porphyrogenita 226

Maria Kantakuzenina 80

Maria die Jüngere 18

Marianos, patrikios 120

Marinus 1, 2, 7, 10

Maskulinität 366, 370, 371, 373, 375

Mathematik 296

Matthaios von Ephesos 437

Matthaios Kantakuzenos 437

Matthaios Laskaris Palaiologos 99

Mauch, Carl 204

Mauropus $\rightarrow$ Ioannes

Maximos Planudes 435, 437, 438, 440, 442,444 
Maximos Scholastikos 176

Mazaris 96, 97, 100, 278, 283, 442

Medea 464, 471, 472

Medeia 101

Meer 387, 389

megas domestikos 73-81, 84, 85, 89, 94, 102

megas drungarios 95

megas dux 74, 75, 93

megas hetaireiarches 77

megas logothetes 97-99, 441

Meinung, persönliche 443

Mekka 206

Melas 230, 232

Melodos $\rightarrow$ Kosmas

Menander Rhetor 54, 55, 141, 144, 147, $149,153,155,263,272,378,383$, 433-436

Mercurius, hl. 5, 9

mesazon 78, 79, 85, 98, 441

Mesopotamites $\rightarrow$ Konstantinos

Metaekphrasis 472

Metanoeite $\rightarrow$ Nikon

Metapher 155, 384, 386-389, 390, 391, 400

Metatext 143

Methodios I., Patriarch 381, 388, 404

Metochites $\rightarrow$ Theodoros

Metrophanes 381, 382

Meyersches Gesetz 315

Michael I. 158

Michael III. 220

Michael VII. 297

Michael VIII. Palaiologos 71, 75, 79, 88, $123,435,444$

Michael IX. 71, 437, 444

Michael Apostoles 440, 447

Michael Attaleiates 3, 100, 109

Michael Burtzes 130

Michael Choniates 229, 250

Michael Gabras 279, 438, 439, 446

Michael Glykas 5, 7

Michael Haplucheir 280, 281

Michael Italikos 283
Michael Palaiologos 230

Michael Panaretos 101

Michael Pantechnes 416

Michael Psellos 3, 4, 6, 7, 19, 21-23, 107, $115,135,137,211-215,218-223$, 257, 263, 287, 293-298, 299, 302, $358,411-416$

Michael von Thessalonike 177

Militärrhetorik 111

Mimesis 141, 143, 358, 360, 371, 374, 386

Minimus $\rightarrow$ Basilius

minsoi 77

Mistras 100, 101

Mitkaiser 213

Mönchsformel 404

moicheianischer Streit 13, 16

Monachos $\rightarrow$ Georgios

Monasteriotes $\rightarrow$ Leon

Monodie 100, 138, 141, 313, 314, 320, 334

Monomachos $\rightarrow$ Konstantinos

Morosini $\rightarrow$ Thomas

Mosaik 227, 469

Moschos $\rightarrow$ Ioannes

Moses 288, 400

Motiv 147, 150, 155, 158, 197, 295, 411

Musik 296

Musterautor 413

Mutter 16, 17, 21, 24, 25

Muzalon $\rightarrow$ Georgios

Myrmeleontidae 255

Myrrha 359-374

Mythos 11, 375

narratio $378-380,382$

Narses 123

Natalia 20, 48

Natur 150, 151

Nausikaa 224, 225

Nebridios 59

Neid 122, 138, 192

Neophytos Momitzilas Prodromenos 97

Neoptolemos 464

Nero 220 
Nestor 272

Nestorius 160

Neues Testament 288

Neuplatonismus 293, 296

Nikaia 150, 440

Nikephoros I. 132, 158

Nikephoros II. Phokas 111, 113, 114, 115, $118,119,120,121,122-124,125$, $126,127,129,130,132,134,136$

Nikephoros, Abt 24

Nikephoros, Patriarch 394

Nikephoros Basilakes 357-360, 364-375

Nikephoros Blemmydes 87, 120

Nikephoros Chumnos 96, 281, 300, 301, 4355, 440, 442, 447

Nikephoros Dukas Angelos 80

Nikephoros Gregoras 100, 223, 256, 279 , 302, 440, 441, 447

Nikephoros Pastilas 115

Niketas 18

Niketas Chalkutzes 125

Niketas Choniates 87, 223, 225, 228, 229

Niketas von Medikion 390, 394, 399

Nikias 133

Nikobulos 416

Nikolaos I. Mystikos 259, 261, 264-266, 272, 274

Nikolaos Kabasilas 166, 437, 438, 440

Nikolaos Kallikles 414

Nikolaos Lampenos 440

Nikolaos von Myra 463, 467, 470-472

Nikolaos Studites 384

Nikomedeia 3, 57, 141, 142, 144, 145, $147,150,153,155$

Nikomedes 148

Nikon Metanoeite 391

Nil 206

nomophylax 296

Norm, rhetorische 381

Notaras $\rightarrow$ Lukas

Nothos $\rightarrow$ Basileios

Nus 294

Ochse 240
Odysseus 290

Olearius, Adam 207

Olive 233

Olympia 396

Onomatopoesie 425

Ophir 204

Ophrydas 296

Opsarologos 88

Optativ 265

Orakel 8

Orestes 10

Originalität 383

Orpheus 4, 369, 375

Ostafrika 203

Osterfest 132

Ovid 359, 363, 368-373, 375, 376

Pachymeres $\rightarrow$ Georgios

Palaiologina $\rightarrow$ Helena, Theodora

Palaiologos $\rightarrow$ Andronikos III., Ioannes V., Konstantinos, Manuel, Michael

Palästina 207, 247

Palast 445

Palimpsest 100

Pan 361

Panaretos $\rightarrow$ Michael

Pandaisia 389, 390

Panegyrik 445

panegyrikos 56

panhypersebastos 74-77, 79, 80, 91

Pantechnes $\rightarrow$ Michael

Paränese (parainesis) 117, 443

Paragraph 379

Parakoimomenos $\rightarrow$ Leon

Parasit 345

Paratext 143, 144

Pardos $\rightarrow$ Gregorios

Paris 207

Parisinus graecus 1517: 160-164, 166

Parisinus graecus 2991A: 99, 100

Parisosis 395

Parmenios 8

Paromoiosis 393, 395

Paronomasie 114, 127, 244, 392, 393, 394 
Pastilas $\rightarrow$ Nikephoros

pathos 61

Patria Konstantinopoleos 103

Patrikios Kalokyros

Paulos, Patriarch 398

Pausanias 144

Peloponnes 96, 100, 440

Pelusium 205

Pera 1

Performanz 24, 281, 284, 362, 365, 367 , $368,370,371,373,374,376,429$, 430, 432, 436, 439, 441, 444, 445, 447

Periander von Korinth 231

Perikles 290

peroratio 191

Perser 422

Perserfeldzug 61

Persien 207, 471

Pest 205

Petros von Atroa 395, 396

Petschenegen 255

Pferd 90, 134, 135, 214, 220, 241, 337

Pferd, weißes 129, 135

Pferderennen 61, 64, 125

Pferdestatue 471, 473

Pfirsich 249

Pherekrates 244

Philadelphia 440

Philaretos 18, 377

Philipp von Makedonien 469, 473

Philippa 229, 234

Philippopolis 372

Philon 335, 336, 340

Philosophos $\rightarrow$ Ioseph

Philostorgios 410

Philostratos 142, 464, 465, 470, 473

Philotheos 261, 262, 264, 266, 267, 270, 274

Phineus 235

Phoenix 248

Phoinissai 472

Phokas $\rightarrow$ Bardas, Leon

Phokier 473
Phokis 469

Phonem 424, 425

Photeinos 21

Photios 172, 265, 277, 403

Pisides $\rightarrow$ Georgios

Planudes $\rightarrow$ Maximos

Platon 6, 144, 279, 288, 291-294, 299$301,335,336,342,346,347,396$, 412,413

Platon, Onkel Theodoros' Studites 16, 17, $19,20,22,24,157,393,399,405$

Platonismus 295

Plotin 336

Plutarch 335, 336, 340

Poesie 358

Polybios 114

Polyeuktos, Patriarch

Polyneikes 464, 472

Polyptoton 154, 392

Polyxena 464, 474

Popularphilosophie 190

Porikologos 88, 93-95, 97, 99

Poseidon 145, 147, 148, 154, 362

Potidaia 322

Prätext 142, 144

Preslav 135

primikerios 77

Priscilla 20, 48

Prodromu-Petra Kloster 100

Progymnasma, progymnasmata 357-360, $367,371,372,463,466-468,470$

Prooimion 302, 436

Prokataskeue 413

Proklos 1-7, 9-11

Prokopios von Gaza 359

Proklos von Konstantinopel 179

Prokopios von Kaisareia 112, 117, 130, 136, 138, 139, 204, 205, 471, 472

prokypsis 71, 72, 82

Prometheus 464, 465, 471, 472

Pronoia 114

Prooimion 90, 107, 111, 114, 137, 144, 145, 147, 302, 377-386, 388, 391, 393-396, 398-400, 402-407, 436 
Prosopopoiie 112

prosphonetikos $65,435,438$

protobestiarios 74, 75, 77, 79-91, 94

protonobelissimos 94

protostrator 94

Prusa 232, 236

Psellos $\rightarrow$ Michael

Pseudo-Hermogenes 463, 468

Pseuod-Kallisthenes 336

Pseudo-Kodinos 70-74, 79-83, 84-86, 97-89, 91-93, 95, 97-101, 102

Pseudo-Nonnos 410

Pseudo-Quintilian 189

Psogos 371

Psyche 358

Ptochoprodromos 280, 414

Publikum 24, 55, 108, 404

Publikumsapostrophe 58, 61, 63

Pulologos 88, 90, 91, 102

Purpur 90, 92, 121

Pylades 10

Quintilian 54, 189, 282, 466, 470

Rede 130, 131, 134, 136, 137, 157, 190$192,200,221,267,363,371$

Rede, Vortrag 78

Redepause 133

Redner 147, 151-153, 191, 196, 198, 199, $262,267,269,274,367,368,419$, 425, 428, 432, 438, 440, 443, 445, 467 s.a. Rhetor

Reicher 190, 191, 193, 196-199

Reim 198, 422, 428 s.a. Binnenreim, Endreim

Reiter 130, 131, 135

Religionspolitik 59

Renier von Montferrat 241

Residuen 311, 312, 317, 318, 321-325, 341-343, 345

Residuenanalyse 333

Rhakendytes $\rightarrow$ Ioseph

Rhaul $\rightarrow$ Emmanuel

Rhein 58
Rhetor 269, 412, 413, 428 s.a. Redner

Rhetorenedikt (362)

Rhetorik 59, 62, 65, 372, 383, 403, 407, 465

Rhetoriklehrer 190

Rhetorikschule 65

rhetorische Frage 307, 309, 317, 345

rhetorische Situation 433

Rhodopen 131

Rhodos 142, 152

Rhythmus 419, 425-427

Richter 199

Ringkampf 396

Ringkämpfer 387, 396

Ringkomposition 62

Roger II. 244

Rom 469

Roman 137

Romanos II. 116, 119, 120

Romanos III. 219

Rotes Meer 205, 206

Rufinus von Aquileia 409

Rus 131, 132

Ruth 20, 21

Sakkoudion 20, 45, 46, 157

Salomon 204, 288

Samuel 20

Sarazenen 259, 263, 271

Sardianos $\rightarrow$ Ioannes

Sattel 89

Satzlänge 306, 313, 316, 317

Satzschluß 342, 343

Schauspiel 125

Scheffel 399

Schiffsmetapher 387-389

Schimmel 135, s.a. Pferd

Schlafen 70

Schlange 233, 237, 249-252, 256, 424, 425

Scholarios $\rightarrow$ Georgios

Scholastikos $\rightarrow$ Maximos

Schriftstellerei 388

Schüler 288, 292 
Schulbesuch 292

Schulbuch 100

Schwan 247

Schwarzes Meer 131, 440

Schweigen 386, 399, 404, 436

Schwein 240

Schwert 115

Scipio Carteromachus 413

sebastokrator 75,78

sebastos 74

Seefahrt 197

Selbstdarstellung 23

Selbstvariation 256, 257

Selymbria 71

Sentenz 400, 401

Sergios, Patriarch 422

Sermo humilis 288

Seth Skleros 249

Seuche 16

Sextus Iulius Africanus 107

Sguros $\rightarrow$ Leon

Siculus $\rightarrow$ Diodorus

Sieg 135

Sikeliotes $\rightarrow$ Ioannes

Sikidites 232

Silbe 421, 427

silention 267

Simbabwe 206

Simon magus 10

Sizilianer 234, 246, 249, 256

Sizilien 126, 132

Skleros $\rightarrow$ Bardas, Seth

Skulptur 464, 472

Skylitzes $\rightarrow$ Ioannes

Smyrna (s. a. Myrrha) 142, 144, 145, 153

Socken 89,90

Sokrates 254, 291

Solon 290

Sonne 198, 282

Sonnenfinsternis 127, 205

Sopatros 328

Sophronios von Jerusalem 170, 172, 179

Sparta 220

Spiel 229
Spielleidenschaft 221

Spinne 255

Spion 131

Sprachstatistik 306

Sprecher 196, 368 s. a. Redner

Sprecherwechsel 196

Sprichwort 395

Spyridonakis $\rightarrow$ Ioannes

Stadtbeschreibung 148, 149, 151

Stadtlob 148-150

Standardabweichung 311

Standardnormalverteilung 307, 311, 314

Statistik 306, 329, 342, 349

Statue 87, 149, 150, 224, 225, 240, 467, $468,470-474$

Stenographie 221

Stephan Nemanja 237

Stephanos Hagiochristophorites 247

Stephanos Kontostephanos 235

Stephanos der Jüngere 18

Stephanos, Patriarch 266, 273

Steuereintreiber 231

Stheneboia 10

Stiefel, kaiserliche 89, 121

Stier 239

Stil 109, 153-155, 269, 300, 391, 426, 435

Stilicho 54

Stilmittel 384, 391-393

Stimme 113

Straßburg 57, 61-63, 65

Studioskloster 20

Studites $\rightarrow$ Nikolaos, Theodoros

Suda 414, 474

Süleyman 207

Sulphur 2, 3, 7, 9

Sumatra 205

Svjatoslav Zgorević 129, 134, 135, 136

Sycae 2

Symeon Magistros 236

Symeon Neos Theologos 358

Symmachos 336

Synadene $\rightarrow$ Theodora

Synaxarvers 391

Synesios von Kyrene 278, 416, 417 
Synkrisis $61,64,90,368$

Syntaxanalyse 306

Syrianos 6, 328

Syrien 65

Talisman 8

Tanz 220

Tarasios 16, 388, 404, 405

Tarsener 116

Tarsos 125, 126

Taufe 164, 165

Theater 64, 151, 218, 220, 277, 278, 370, 440,467

Theatralik 372

Theatron ( $\theta \dot{\alpha} \alpha \tau \varrho o v)$ 91, 96, 97, 113, $277-$ $279,282,357,429-431,440,441$

Thebai 152, 231, 472

Theias 359, 361, 365-368

Theodora 297

Theodora (Tochter von Ioannes Kantakuzenos) 71

Theodora Palaiologina 50

Theodora Synadene 50

Theodora von Thessalonike 18

Theodoros II. Laskaris 411

Theodoros Gazes 100

Theodoros Graptos 181

Theodoros Hyrtakenos 440, 441, 447

Theodoros Metochites 97, 100, 299, 300302, 440, 447

Theodoros Prodromos 359, 364

Theodoros Studites 13-15, 17, 22-25, $399,405,411$

Theodosios 10

Theodosios Boradiotes 245, 249

Theodote, Mutter des Michael Psellos

Theognis 290

Theokritos 238

Theoktiste, Mutter des Theodoros Studites 13-18, 20-22, 24, 25, 42, 46

Theon von Alexandria 9, 139, 463

Theon von Samos 470

Theophilos Korydaleus 415, 417

Theophylaktos von Ochrid 279, 416
Thessalonike 47, 80, 157, 165, 166, 242, $247,256,438,440$

Thomaïs von Lesbos 18

Thomas Magistros 78, 300

Thomas Morosini 231, 241

Thoros 235

Thrasybulos 231

Threnos 151-153, 398

Thukydides 110, 111, 112, 113, 137, 144, 330,344

Tigris 61

Timon 321

Tischrede 268

Toniki 205

Topik, Topos, Topoi 17, 24, 54, 56, 57, 123, 144, 147, 148, 149, 190, 200, 268, 321, 381 383-389, 391, 399, 400-407, 445

topisches Schema 400-402

Tradition 391

Transtextualität 143

Trapezunt 74, 101

Traumdeuter 8

Triklinon 269-271, 273

Trisagion 6, 10

Triumph 119, 441

Troia 134, 152, 464, 465, 473

Trompete 91

Troparion 176, 177

Tsunami 152

Türken 71, 231, 233-235, 252, 419, 420

Türkenherrschaft 417

Tugend 382, 386

Tullius Laurea 335

tu Narsu 298, 299

Tyche 321

Typikon 50

Tyrannis 123

Tzephre 91

Tzetzes $\rightarrow$ Ioannes

Umgangsformen 60

Ungarn 232, 251, 252

Urhan 71 
Variatio 307, 394, 420

Vaticanus Barberinus graecus 64: 101

Vaticanus graecus 162: 101 975: 101, 102

Venezianer 234, 426

Vergessen 398, 399

Vergiftung 137, 214

Vergiftungstod 198, 199

Vergil 197, 200

Veritas-Formel 404

Verres 470

Verschwörung 211

Vision 8

Vitalianus 1, 2, 9, 10, 11

Vogel 91, 245

Vokabularreichtum 307, 337

Vokabularumfang 309, 311, 313, 327, 348

Vortrag 59, 113, 446, s.a. Rede

Vortragssituation 59

Vulkanausbruch 205

Wachs 254

Wahrscheinlichkeitsaussage 306

Wal 95, 244

Waräger 135, 218

Wasser 230

Wasserversorgung 135

Weihnachten 77, 79, 81, 88, 93, 227, 266, 437

Weiß 70, 89, 225, 423

Weltchronistik 107

Wespe 254

Wettkampf 442

Widmungsapostrophe 62

Wien 207
Wilhelm von Montferrat 224

Wortartenverteilung 323, 324

Wortlänge 307, 314

Wortley Montagu, Mary 207, 208

Xenophon 335, 342, 346

Xiphilinos $\rightarrow$ Ioannes

Yahya von Antiocheia 219

z-Wert 307, 309, 311-314, 316, 318-320, 325,349

Zacharias $\rightarrow$ Ioannes

Zauberer 8

Zeichen 470

Zeit 147, 404, 473

Zelt 246

Zensur 293

Zeremoniell 260 s.a. Krönung

Zeremonienbuch 86

Zeugminon 251

Zeus 228, 362, 363, 366

Zigabenos $\rightarrow$ Euthymios

Zitat 395, 396, 400

Zoe, Kaiserin 219

Zoe Karbonopsina 274

Zoroaster 4

Zuhörer 109, 112, 115, 119, 121, 133, 137, $282,283,365,368,371,390,395$, 403, 425, 432, 433, 440, 443, 445, 448, 466, 467, 470, 472, 473 s. a. Auditorium, Hörer, Hörerschaft

Zunge 247, 254, 388, 389, 394

Zuschauerrolle 220

Župan 238, 249

Zypern 125 